\title{
Gênero e esporte na historiografia brasileira: balanços e potencialidades
}

\author{
Silvana Vilodre Goellner[1]
}

\begin{abstract}
Resumo
Este texto analisou a emergência da categoria gênero como ferramenta analítica, cuja utilização possibilitou a ampliação de temas, objetos e problemas na produção acadêmica do campo da história do esporte. Foi desenvolvido um breve balanço desta produção, bem como foi apontado à existência de temáticas e ferramentas analíticas ainda pouco investigadas, sobretudo, nos estudos desenvolvidos no Brasil.
\end{abstract}

Palavras-chave: gênero; esporte; história.

\section{Género y deporte en la historiografía brasileña: equilibrios y potencialidades}

\section{Resumen}

Este texto analizó la emergencia de la categoría género como herramienta analítica, cuya utilización posibilitó la ampliación de temas, objetos y problemas en la producción académica del campo de la historia del deporte. Fue desarrollado un breve diagnostico de esta producción, bien como fue apuntado a la existencia de temáticas y herramientas analíticas aun poco investigadas, sobre todo, en los estudios desarrollados en Brasil.

Palabras clave: genero; deporte; historia.

\section{Gender and sports in the Brazilian historiography: balance and potentials}

\begin{abstract}
This paper analyzed the emergence of the gender category as an analytical tool whose use has enabled the expansion of subjects, objects, and problems in the academic production of sport historiography field. It developed a brief review of that production, as well as pointed to the existence of thematic and analytical tools that are still poorly investigated, mainly in studies performed in Brazil.
\end{abstract}

Keywords: gender; sport; history.

\section{Genre et sport dans l'historiographie brésilienne : enquête et potentiel}

\section{Résumé}

Ce texte retrace l'émergence du " genre » comme catégorie d'analyse dont l'emploi a permis lélargissement du champ de l'historiographie du sport à de nouveaux thèmes, objets et problèmes. Il fait un bilan de cette production académique et indique des thématiques et des outils d'analyse qui demeurent toujours peu investigués au Brésil.

Mots-clés: genre; sport; histoire. 
pesquisa sobre gênero no âmbito da história do esporte tem se constituído, desde meados da década de 1970, como uma possibilidade investigativa de diversos autores em diferentes contextos culturais, especialmente na Europa e nos Estados Unidos. No Brasil, sua maior disseminação ocorreu nos anos 1980, inicialmente por meio de pesquisas desenvolvidas em programas de pós-graduação, fundamentalmente na área da educação.

Mais do que esboçar o estado da arte dessa produção, vale ressaltar alguns movimentos cujos desdobramentos acadêmicos e políticos possibilitaram a emergência de novas abordagens no campo da história do esporte, ao contemplarem objetos, problemas, instrumentos analíticos e fontes até então pouco analisados. Nessa direção, destaca-se a contribuição do movimento feminista $^{1}$ das décadas de 1960 e 1970, o qual também promoveu a elaboração de aportes teórico-epistemológicos capazes de subsidiar práticas e discursos que buscavam valorizar a experiência feminina que era comumente negligenciada. O campo acadêmico denominado História das Mulheres ${ }^{2}$ decorre de tal movimento e intencionalidade.

Foi nesses anos que ocorreu o registro, na história, de grupos anteriormente "silenciosos" definidos por diferenças de raça, sexo, preferências sexuais etc.

Na década de 1960, muitas [...] questões foram bruscamente trazidas à tona, quando o político e o estético se fundiram na chamada contracultura [...]. Os negros e as feministas, os etnicistas e os gays, as culturas nativa e do "terceiro mundo" não formam movimentos monolíticos, mas constituem diversidade de reações a uma situação de marginalidade e excentricidade percebida por todos. ${ }^{3}$

A produção historiográfica, sobretudo americana e francesa, desenhou os primeiros contornos dos estudos acerca das mulheres demarcando um território acadêmico e político necessário à desejada busca pela visibilidade. Esse cenário sofreu uma alteração com a publicação do ensaio Gender: a useful category of historical analysis, ${ }^{4}$ escrito pela historiadora norte-americana Joan Scott. Nele, a autora propôs a utilização do gênero como uma ferramenta

'O movimento feminista não pode ser entendido como um bloco homogêneo. Desde suas origens, apresentou uma série de nuances e dissonâncias, e o próprio termo feminismo não é unívoco. Autoras como Janet R. Richards (1994) e Judith Evans (1995) analisaram e classificaram diferentes grupos e vertentes. Richards identificou duas grandes vertentes: o feminismo liberal e o radical. Evans apresentou uma rede mais ampla de orientação com realce para as vertentes liberais, radicais, culturais, socialistas e pós-modernas. Ana Macedo e Ana Luisa Amaral, Dicionário da crítica feminista, Porto, Edições Afrontamento, 2005.

${ }^{2}$ Algumas historiadoras feministas cunharam uma nomenclatura particular, dentro do campo dos Women Studies: o termo Herstory que, inicialmente, foi utilizado para chamar a atenção ao uso universal do masculino genérico subtendido na History (his-story). A motivação para esta crítica fundamenta-se no desajustamento entre a linguagem e a realidade a que esta se refere, o que acaba por omitir o papel desempenhado pelas mulheres como sujeitos sociais na hstória. Ao propor a utilização do termo Herstory, as mesmas pretendem conferir às mulheres um lugar e, ao mesmo tempo, devolver a história às mulheres na medida em que este termo refere-se à teorização e ao registro da experiência, da vida e da linguagem delas. Maggie Humm, The Dictionary of Feminist Theory, Columbus, Ohio State University Press, 1996, p. 35. Sobre esse tema ler: Ana Macedo e Ana Luisa Amaral, op. cit., p. 23.

${ }^{3}$ Linda Hutcheon, Poética do Pós-Modernismo, Rio de Janeiro, Imago, 1991, p. 32.

${ }^{4}$ Publicado em 1986, este ensaio foi traduzido pela revista Educação \& Realidade em 1990 a partir de uma versão francesa. Em 1995, o mesmo periódico veiculou nova versão traduzida do texto original em inglês. Joan Scott, "Gênero: uma categoria útil de análise histórica", Educação \& Realidade, vol. 20, n. 2, Porto Alegre, 1995, p. 71-99. 
analítica, deslocando, desse modo, a centralidade na categoria identitária mulher para o caráter relacional entre os sexos, enfatizando "as conotações sociais do gênero em contraste com as conotações físicas do sexo".

Embora seja consensual a recusa à ideia de que o sexo anatômico é determinante na imposição das diferenças entre homens e mulheres - isto é, de que essas identidades não são biologicamente dadas, mas construídas social e culturalmente -, o termo gênero não foi assumido de forma homogênea na teorização feminista, ${ }^{6}$ inclusive nos estudos de cunho historiográfico.

Inicialmente, sua utilização motivou estudos que se propunham a denunciar a quase invisibilidade das mulheres como sujeitos tanto nas grandes narrativas sobre a história da humanidade, quanto como protagonistas na própria produção historiográfica. Tais estudos reivindicaram à história a tarefa de salientar a importância da participação das mulheres na constituição das sociedades e culturas, reconhecê-las nos espaços público e político e nos meandros do cotidiano. Permitiram ainda que, na produção acadêmica da história do esporte, em especial nos países de língua inglesa, a categoria gênero fosse agregada às análises empreendidas. Introduziram, assim, a asserção de que o esporte é uma prática social não apenas sexuada como também genérica: ${ }^{7}$ sexuada, pois pessoas de ambos os sexos o praticam; generificada, porque nele se constroem e expressam identidades masculinas e femininas. ${ }^{8}$

Tais estudos inauguraram a identificação do esporte como um importante espaço na construção e incorporação de masculinidades e feminilidades. ${ }^{9}$ Além disso, romperam com a naturalizada aceitação de que as diferentes experiências esportivas vividas por homens e mulheres justificavam-se pela diferente biologia de seus corpos.

\section{Histórias do esporte: o gênero como uma categoria analítica}

A inserção de aportes teórico-epistemológicos advindos dos feminismos no campo da pesquisa historiográfica possibilitou o surgimento de objetos de investigação outrora interditos, muitos deles analisados a partir das relações de gênero. Se por um lado essa inserção fomentou uma vasta produção sobre

\footnotetext{
$\overline{5}$ Joan Scott, "O enigma da igualdade", Revista Estudos Feministas, vol. 13, n. 1, Florianópolis, 2005, p. 11-30. "Segundo Linda Nicholson, embora a palavra gênero tenha um significado bastante claro para o feminismo, ela é usada de duas maneiras diferentes e até certo ponto contraditórias. De um lado, é sempre usado em oposição a sexo para descrever o que é socialmente construído em oposição ao que é biologicamente dado. Essa compreensão é mais visível nas primeiras teorizações feministas. De outro, tem sido cada vez mais usado como referência a qualquer construção social que tenha a ver com a distinção masculino/ feminino, incluindo as construções que separam corpos femininos dos masculinos. Aqui há a percepção “de que a sociedade forma não apenas a personalidade e o comportamento mas também as maneiras como o corpo aparece". Linda Nicholson, "Interpretando Gênero", Revista Estudos Feministas, vol. 8, n. 2, Florianópolis, 2000, p. 9-42. Sobre tal tema ler também: Verena Stolke, "La mujer es puro cuento: la cultura del gênero", Revista Estudos Feministas, vol. 12, n. 2, Florianópolis, 2004, p. 77-105; Silvana Aparecida Mariano, "O sujeito do feminismo e o pós-estruturalismo", Revista Estudos Feministas, vol. 13, n. 3, Florianópolis, 2005, p. 483-505. 7Jennifer Hargreaves, "The Social Production of Gender Through Sport", Theory, Culture and Society, vol. 3, n. 1, 1986, London, p. 114-119; Ian Wellard, "Able bodies and sport participation: social construction of physical ability for gendered and sexually identified bodies", Sport, Education and Society, vol. 11, n. 2, p. 105-119; Michel Messner, "Sports and Male Domination: The Female Athlete as Contested Ideological Terrain", In: Susan Birrell; Cheryl Cole (eds.), Women, Sport, and Culture, Champaign, IL, Human Kinetics, 1994, p. 94-112.

spaula Silva, A construção/estruturação do género na educação física, Lisboa, Comissão para a Igualdade e para os Direitos das Mulheres, 2007.

${ }^{9}$ Nancy Theberge, "Toward a Feminist Alternative to Sport as a Male Preserve", In: Susan Birrell, Cheryl Cole (eds.), Women, Sport, and Culture, Champaign, IL, Human Kinetics, 1994, p. 181-192.
} 
a construção de masculinidades e feminilidades em vários campos acadêmicos, por outro se fez restrita no âmbito específico da história do esporte.

Tal afirmação pode ser identificada na ínfima atenção que os feminismos têm dado a esta prática corporal, ${ }^{10}$ especialmente na produção acadêmica de língua portuguesa. ${ }^{11}$ Do mesmo modo, os estudos a respeito do esporte, inclusive os de cunho historiográfico, em sua maioria, têm ignorado a própria generificação que o estrutura. ${ }^{12}$

Em que pese essa dupla marginalidade, é necessário ressaltar que vários objetos atualmente investigados no campo da história do esporte foram possíveis apenas em função da contribuição advinda dos feminismos e de suas proposições políticas e acadêmicas.

Ao desconstruírem a representação naturalizada de que homens e mulheres formam-se masculinos e femininos devido às diferenças corporais, sendo que as mesmas justificam determinadas desigualdades, atribuem funções sociais e determinam papéis a serem desempenhados por um ou outro

\section{Os corpos, as gestualidades, as representações de saúde, beleza e desempenho são construções históricas}

sexo, os estudos feministas e de gênero possibilitaram outros pontos de vista sobre o esporte. Permitiram, por exemplo, identificar que os corpos, as gestualidades, as representações de saúde, beleza e desempenho são construções históricas, as quais, em diferentes tempos e culturas, foram associadas aos homens e/ou às mulheres. Tornaram visíveis os processos generificadores constituintes do esporte, ao ressaltarem que são produzidos e reproduzidos nele masculinidades e feminilidades e que estas são sempre históricas, mutantes e provisórias. ${ }^{13}$

Foram, portanto, determinantes para desconstruir muitos discursos e práticas que afirmavam hierarquias e poderes ${ }^{14}$ de determinados indivíduos e grupos. Com isso, evidenciou-se que a naturalizada aceitação do esporte como um campo de "reserva masculina" ${ }^{15}$ justifica-se não pela distinta biologia dos corpos de homens e mulheres, mas por aspectos sociais, culturais e históricos.

\footnotetext{
${ }^{10}$ Jennifer Hargreaves, Sporting females: critical issues in the History and Sociology of women's sports, London, Routledge, 1994; Anne Flintoff, Sheila Scraton, "Feminist research and Physical Education”, In: Andrew Sparkes (ed.), Research in Physical Education and Sport Exploring Alternative Visions, London, Falmer Press, 1992, p. 167187; Ann Hall, Feminism and Sporting Bodies Essays or Theory and Practice, Illinois, Human Kinetics, 1996. "Paula Botelho-Gomes, Paula Silva, Paula Queirós, Equidade na educação. educação física e desporto na escola, Lisboa, Associação Portuguesa Mulher e Desporto, 2000; Paula Silva, Paula Botelho-Gomes, Silvana Vilodre Goellner, "Masculinities and sport: the emphasis on hegemonic masculinity in Portuguese physical education classes", International Journal of Qualitative Studies in Education, vol. 25, 2011, p. 1-23.

${ }^{12}$ Paula Silva, A construção/estruturação do género na educação física, Lisboa, Comissão para a Igualdade e para os Direitos das Mulheres, 2007.

13Silvana Vilodre Goellner, "Feminismos, mulheres e esportes: questões epistemológicas sobre o fazer historiográfico", Movimento, vol. 13, n. 2, Porto Alegre, 2007, p. 123-135.

${ }^{14} \mathrm{O}$ poder, aqui, é tomado a partir da teorização de Michel Foucault, que o percebe como difuso, descentralizado e horizontal. Michel Foucault, Microfísica do Poder, Rio de Janeiro, Graal, 1992.

${ }^{15}$ Eric Dunning, "O desporto como área masculina reservada: notas sobre os fundamentos sociais da identidade masculina e as suas transformações”, In: Norbert Elias, A busca da excitação, Lisboa, Diefel, 1992, p. 145-156.
} 
Em decorrência de formulações semelhantes à mencionada, os feminismos reclamaram às mulheres a sua condição de sujeito no esporte, analisando-o como um espaço político e, consequentemente, um lugar de resistência e transformação das relações de gênero. ${ }^{16}$

No campo da pesquisa historiográfica brasileira, o primeiro enfoque investigativo recaiu sobre as mulheres. Tal produção, revestida de caráter político, colocou em suspeita a representação da fragilidade feminina, descobrindo-se que, desde os primórdios do esporte, elas se fizeram presentes em diferentes dimensões dessa prática cultural, apesar da ausência de registro na oficialidade dos discursos.

Decorrentes e complementares a este foco, foram despontadas investigações sobre feminilidades e, em quantidade infinitamente menor, acerca das masculinidades. Duas vertentes podem ser identificadas nessa direção: uma cujos estudos reafirmam as categorias identitárias homem e mulher, anulando as diferenças presentes em cada polo dessa representação; outra que, na contramão, enfatiza as divergências inerentes a cada uma dessas categorias binárias, evidenciando, portanto, a impossibilidade de se generalizar a mulher ou o homem.

A primeira delas, ao universalizar as representações de mulher e homem, atribuindo-lhes características distintas, inatas e imutáveis, sugere que a partir dessas características podem ser determinados os traços de caráter e comportamento, funções sociais, espaços de pertencimento e possibilidades de socialização para todos. Operam com o conceito de estereótipo a partir do qual modelos de masculinidade e feminilidade são construídos, tendo como base o sexo biológico. Nessa direção, as pesquisas empreendidas pouco problematizaram a diferente inserção, adesão e permanência de homens e mulheres no universo cultural do esporte, reafirmando, de certo modo, a representação de que para eles destina-se a aventura, a potência, o desafio, a força; para elas, a aventura comedida, a potência controlada, a força mensurada e o desafio ameno. ${ }^{17}$

Já a segunda vertente aponta que marcadores sociais - por exemplo, raça/etnia, geração, sexualidade, religião, classe social etc. — provocam distinções entre mulheres e homens, o que implica aceitar a diversidade dos modos de ser de um e de outro polo dessa relação. Essa assunção está presente em pesquisas que focalizam a participação de mulheres em esportes representados como masculinos e na participação de homens naqueles considerados femininos, apontando que nos mesmos são formados modos plurais de viver e produzir feminilidades e masculinidades.

Cabe destacar que grande parte da produção inaugural brasileira sobre esporte e gênero manteve estreita relação com a historiografia da educação, cujo lócus investigativo teve como principal foco a Educação Física escolar. Tais estudos conferiram visibilidade à temática, desencadeando um movimento no interior do campo acadêmico da Educação Física e das Ciências do Esporte. Se, por um lado, estas já apresentam uma trajetória reconhecida dos estudos de gênero, por outro, ainda carecem de maior investimento, pois algumas temáticas e metodologias ainda encontram-se a descoberto.

\footnotetext{
${ }^{16}$ Ann Hall, "From pre to postfeminism: a four-decade journey", In: Pikko Markula (org), Feminist sport studies: sharing experiences of joy and pain, New York, State University of New York Press, 2005, p. 56-67.

${ }^{17}$ Silvana Vilodre Goellner, "Entre o sexo, a beleza e a saúde: o esporte e a cultura fitness", Labrys. Estudos Feministas, vol. 10, Brasilia, 2006, p. 6-15.
} 


\section{Gênero e esporte: potencialidades temáticas e analíticas para a historiografia brasileira do esporte}

A inclusão tardia do gênero como uma ferramenta analítica na pesquisa historiográfica acerca do esporte pode sugerir um entendimento tácito de que este representa um campo fortemente associado a pensamentos e ações, que simbolizam o masculino e, como tal, seriam mais resistentes à mudança. ${ }^{18}$ Conforme referenciado, raramente os estudos feministas têm focalizado o esporte como um objeto legítimo e pertinente de investigação, tampouco têm realçado a importância da prática esportiva como uma forma de empoderamento de homens e mulheres. ${ }^{19}$

Ainda que seja possível visualizar uma produção acadêmica orientada nesta direção, os estudos sobre gênero e esportes produzidos no Brasil carecem ainda de uma maior potencialização, quando comparados com a produção internacional da área.

Os estudos sobre masculinidades, por exemplo, ainda são incipientes, e os existentes estão direcionados a uma masculinidade hegemônica, ${ }^{20}$ na qual atributos viris são reconhecidos e incentivados. Estão ausentes análises que apontem para esportes cujo acontecer produz outros modos de ser masculino, por exemplo, as ginásticas rítmica ou aeróbica e a patinação artística, modalidades nas quais o corpo do homem parece ser incapaz de protagonizar uma estética corporal tradicionalmente associada a uma exclusiva prática feminina.

Do mesmo modo, estudos que tematizem questões relacionadas às homossexualidades ainda são pouco visíveis. Impera, no campo da pesquisa historiográfica sobre esporte, o princípio da heteronormatividade, ${ }^{21}$ praticamente invisibilizando os sujeitos que dele escapam.

Nessa direção, uma grande potencialidade para os estudos sobre esporte, observados a partir da sua vertente histórica, reside na incorporação de análises que rompam com o binarismo homem-mulher, feminilidade-masculinidade, heterossexualidade-homossexualidade, uma vez que, mesmo que esses termos sejam usados no plural, ainda não visibilizam os "paradoxos da diferença".22

Desse modo, a teorização queer ${ }^{23}$ tem se revelado uma fecunda possibilidade de ancoragem teórica, na medida em que opera com a desconstrução dos binarismos, inclusive aqueles que estão presentes nos estudos de gênero. Ao eliminarem do horizonte analítico o determinismo biológico e os binaris-

\footnotetext{
${ }^{18}$ Roberta Bennett; Gail Whitaker, Nina Jo Woolley Smith, Anne Sablove, "Changing the rules of the game: reflections toward a feminist analysis of sport”, Women's Studies Int. Forum, vol. 10, n. 4, 1987, p. 369-379. ${ }^{19} \mathrm{Ann}$ Hall, Feminism and sporting bodies essays or theory and practice, Illinois, Human Kinetics, 1996. ${ }^{20}$ Robert William Connell, Masculinities, Beverly, University of California Press, 1996; Miguel Vale de Almeida, Senhores de si: uma representação antropológica da masculinidade, Lisboa, Fim de Século, 1995. ${ }^{21}$ Por heteronormatividade entende-se o sistema social de organização do gênero e da sexualidade que define a heterossexualidade como norma e a homossexualidade como desvio. Miguel Vale de Almeida, A chave do armário: homossexualidade, casamento e família, Florianópolis, Editora da Universidade Federal de Santa Catarina, 2010.

${ }^{22}$ Joan Scott, "O enigma da igualdade", Revista Estudos Feministas, vol. 13, n. 1, Florianópolis, 2005, p. 11-30

${ }^{23} \mathrm{~A}$ palavra queer, de origem inglesa, está relacionada a algo esquisito, estranho ou excêntrico. Durante muito tempo foi utilizada como uma forma de xingamento para sujeitos homossexuais, sendo incorporada pelo movimento gay e lésbico para representar a diferença que não quer ser assimilada ou tolerada. Sobre este tema ler: Guacira Lopes Louro, Um corpo estranho: ensaios sobre sexualidade e teoria queer, Belo Horizonte, Autêntica, 2004
} 
mos, tais estudos favorecem a aceitação da profusão de feminilidades e masculinidades, da permeabilidade entre as fronteiras corporais e a não fixação das identidades. Segundo Judith Butler,

As categorias de identidade nunca são meramente descritivas, mas sempre normativas e, como tal, exclusivistas. Isso não quer dizer que o termo 'mulheres' não deva ser usado, ou que devamos anunciar a morte da categoria. Ao contrário, se o feminismo pressupõe que 'mulheres' designa um campo de diferenças indesignáveis, que não pode ser totalizado ou resumido por uma categoria de identidade descritiva, então o próprio termo se torna um lugar de permanente abertura e re-significação. ${ }^{24}$

Publicado em 2006, o livro Sport, Sexuality and Queer, ${ }^{25}$ organizado por Jayne Dauwell, merece destaque por ser considerado a obra inaugural destas análises. Nele, reúnem-se artigos que buscam refletir sobre o queer no campo do esporte, apontando inclusive para os desdobramento desta teorização nas pesquisas sobre tal prática corporal. ${ }^{26}$ Considerando que o conceito queer pode ser utilizado de diferentes modos a fim de analisar a relação entre esporte e sexualidade, o livro possibilita ainda refletir sobre a fluidez, a multiplicidade e a ambiguidade das identidades sexuais e de gênero presentes no universo do esporte, favorecendo, assim, a emergência de análises ainda pouco abordadas em sua produção historiográfica.

\section{Estudos sobre masculinidades ainda são incipientes, e os existentes estão direcionados a uma masculinidade hegemônica, na qual atributos viris são reconhecidos e incentivados}

Como possibilidade temática, ainda são incipientes estudos que analisem as questões de gênero esportivo na inter-relação com classe social, religião, raça/etnia, sexualidade e geração, o que, de certo modo, indica que as categorias homem e mulher ainda estão prevalentemente circunscritas aos contextos urbano, cristão, branco, heterossexual e jovem.

Investir em tais temáticas implica não apenas uma aproximação acadêmica com a literatura internacional, mas, sobretudo, um posicionamento político, à medida que estudos em tal direção tirarão das zonas de sombra corpos e sujeitos que não podem ser categorizados.

Nesse sentido, a abordagem teórico-metodológica da História Oral pode se constituir em uma ferramenta útil, visto que muitos dos corpos e sujeitos residentes nas zonas de sombra da historiografia do esporte lá estão por não haver registros. Entendida como uma técnica de produção e tratamento de entrevistas, um método de investigação e uma fonte de

\footnotetext{
${ }^{24}$ Judith Butler, Problemas de gênero, Rio de Janeiro, Civilização Brasileira, 2003, p. 22.

${ }^{25}$ Jayne Dauwell, Sport, Sexuality and Queer Theory, London/New York, Routledge, 2006.

${ }^{26}$ Wagner Xavier de Camargo, "Sexualidades, esportes e teoria queer: inter-relações", Revista Estudos Feministas, vol. 13, n. 3, Florianópolis, 2008, p. 1130-1133.
} 
pesquisa, ${ }^{27}$ ao longo das últimas décadas do século XX a História Oral se tornou um dos métodos prediletos de pesquisadoras feministas, as quais a utilizaram por entender que:

[...] nos permite ouvir histórias de indivíduos e grupos que de outra forma seriam ignorados; permite expandir os horizontes do nosso conhecimento sobre o mundo; e estimula o questionamento de nossas próprias hipóteses a respeito das experiências e dos pontos de vista de outras pessoas e culturas. ${ }^{28}$

No entanto, esse entusiasmo não encontrou correspondência na historiografia brasileira do esporte, mesmo quando a categoria gênero figurou como central. Mostrou-se, ainda, como uma possibilidade para, um devir.

\section{Considerações finais}

Os estudos sobre gênero e esporte presentes no campo da história poucas vezes têm evidenciado suas relações com os aportes teóricos advindos dos feminismos. Apesar dessa não explicitação, tais investigações foram possíveis devido à inegável contribuição proporcionada pela teorização feminista, sem a qual a categoria analítica gênero sequer existiria.

As diferentes vertentes epistemológicas do gênero provocaram rupturas na oficialidade de muitos discursos que narravam o esporte, sua emergência e legitimação, sem considerar os processos generificados e generificadores que o estruturam. Por fim, foram imperativas na desconstrução que representa o esporte como um campo naturalmente masculino, ao fazerem ver que, como qualquer outra instância social, esse é um espaço de generificação. Tal fato não ocorre por refletir as desigualdades e diferenciações da sociedade em geral, mas, fundamentalmente, por produzi-las e reproduzi-las.

\footnotetext{
${ }^{27}$ Paul Thompson, A voz do passado: história oral, Rio de Janeiro, Paz e Terra, 1992; Marieta de Moraes Ferreira; Janaína Amado (org.), Usos e abusos da história oral, Rio de Janeiro, Editora da Fundação Getúlio Vargas, 1996. ${ }^{28}$ Daphne Pathai, História oral, feminismo e política, São Paulo, Letra e Voz, 2010, p. 124
} 